\title{
New insights into the interaction of the immune system with non-small cell lung carcinomas
}

\author{
Paul Hofman ${ }^{1,2,3}$ \\ ${ }^{1} \mathrm{CHU}$ Nice, FHU OncoAge, Laboratory of Clinical and Experimental Pathology, Pasteur Hospital, Université Côte d'Azur, Nice, France; ${ }^{2} \mathrm{CNRS}$, \\ INSERM, IRCAN, FHU OncoAge, Team $4,{ }^{3} \mathrm{CHU}$ Nice, FHU OncoAge, Hospital-Integrated Biobank, Université Côte d'Azur, Nice, France \\ Correspondence to: Paul Hofman, MD, PhD. Laboratory of Clinical and Experimental Pathology, Pasteur Hospital, 30 avenue de la voie romaine, \\ BP69, 06001 Nice Cedex 01, France. Email: hofman.p@chu-nice.fr.
}

\begin{abstract}
The basis of current and future lung cancer immunotherapy depends mainly on our knowledge of the molecular mechanisms of interactions between cancer and immune cells (ICs), as well as on interactions occurring between the different populations of intra-tumor ICs. These interactions are very complex, as virtually all immune cell types, including macrophages, neutrophils, mast cells, natural killer (NK) cells, dendritic cells and $\mathrm{T}$ and $\mathrm{B}$ lymphocytes can infiltrate lung cancer tissues at the same time. Moreover these interactions lead to progressive emergence of an imbalance in ICs. Initially ICs have an anti-tumor effect but then induce immune tolerance and eventually tumor progression and dissemination. All the cells of innate and adaptive intra-tumor immunity engage in this progressive phenotypic switch. A majority of non-small cell lung carcinoma (NSCLC) patients do not benefit from the expected positive responses associated with current immunotherapy. Thus, there is urgent need to better understand the different roles of the associated cancer ICs. This review summarizes some of the new insights into this domain, with particular focus on: the myeloid cell population associated with tumors, the tertiary lymphoid structures (TLSs), the role of the P2 purinergic receptors (P2R) and ATP, and the new concept of the "liquid microenvironment" implying blood circulating ICs.
\end{abstract}

Keywords: Non-small cell lung carcinoma (NSCLC); immune cells (ICs); myeloid cells; P2 purinergic receptors (P2R); tertiary lymphoid structure (TLS)

Submitted Jan 24, 2020. Accepted for publication May 18, 2020.

doi: $10.21037 /$ tlcr-20-178

View this article at: http://dx.doi.org/10.21037/tlcr-20-178

\section{Introduction}

The introduction of immunotherapy and its dramatic benefit to overall survival of certain advanced and metastatic non-small cell lung carcinoma (NSCLC) patients has been one of the highlights of this last decade in thoracic oncology. The therapeutic progress observed these last few years has come from a deeper understanding of the basic cellular and molecular mechanisms involved in interactions between cancer cells and the tumor microenvironment (TME), notably immune cells (ICs) (1-3). In this regard, many studies and reviews have been published these last few years concerning the different mechanisms regulating these complex interactions (4-7). More specifically, the development of the different immune checkpoint inhibitors (ICIs) targeting the interaction between PD1 expressed on the T lymphocytes and PD-L1 expressed on tumor cells has increased the knowledge into immunopathology of lung cancer (8-10).

However, despite some major therapeutic breakthroughs leading recently to new international guidelines in treatment of advanced and metastatic lung cancer, many uncertainties persist: (I) some patients are non-responders to ICIs while the tumor cells express high levels of PD-L1; conversely other patients are good responders to ICIs while their tumors do not express PD-L1; and (II) the cellular mechanisms of tumor hyper-progression occurring in certain patients treated with ICIs are not well defined 
A

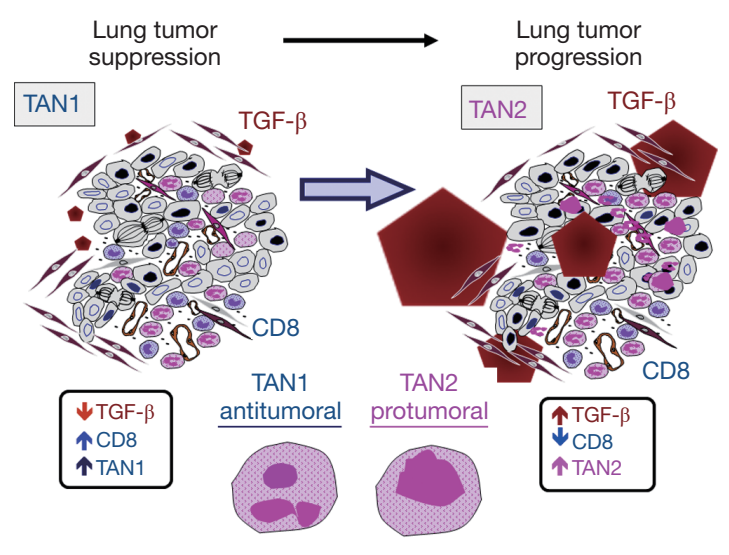

B

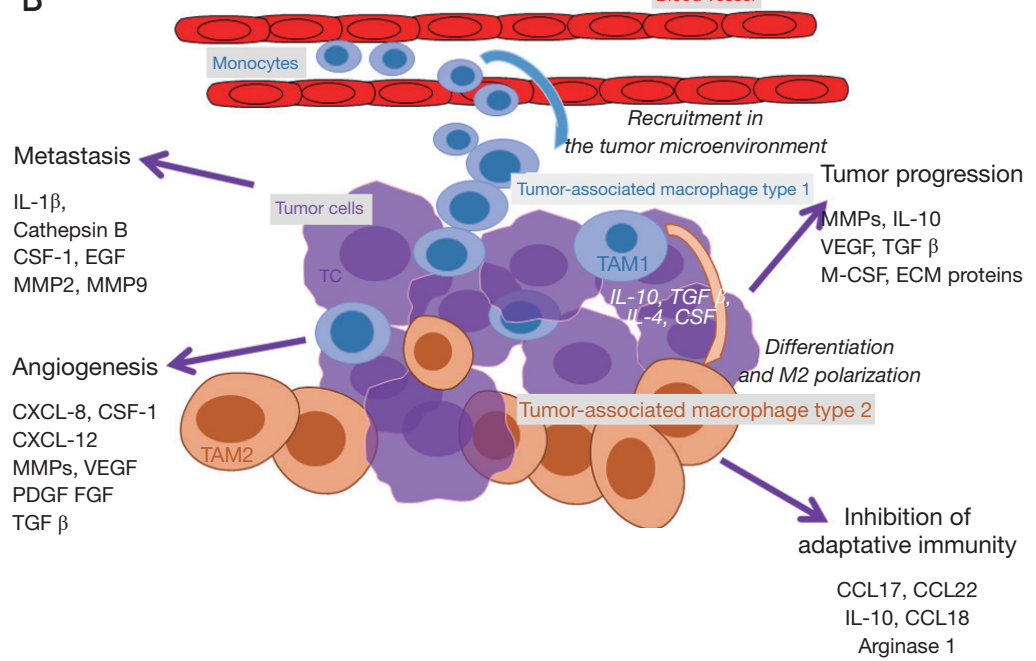

Figure 1 Tumor associated neutrophils (TAN) and tumor associated macrophages (TAM) during tumor progression. (A) Representation of the switch from the TAN type 1 to the TAN type 2. (B) Recruitment of monocytes and macrophages on tumor site and different roles of TAM type 2 during tumor development.

and there is no predictive biomarkers of the tumor hyperprogression until now (11-13).

After briefly reporting the main cellular actors of the innate and adaptive immunity and their respective roles in the interaction between ICs and tumor cells, this review will highlight the recent advances in the domain of tumor cell-ICs interactions. Some specific fields will be discussed including the role of: (I) the different myeloid populations, notably myeloid derived suppressive cells (MDSC); (II) the ATP/ADP and the P2 purinergic receptors (P2R); (III) the blood circulating ICs in metastatic dissemination; and (IV) the involvement of tertiary lymphoid structures (TLSs) in immunotherapy. Finally, some new approaches analyzing the TME in lung cancer as well as some new potential therapeutic strategies targeting the ICs will be discussed.

\section{Intra-tumoral immunity in lung cancer: from immunosurveillance to the status of immunotolerance}

Following an initial phase of immunosurveillance including tumor cell destruction by efficient intra-tumoral ICs, the cancer escapes this surveillance and tumor cells proliferate due to a phase of immunotolerance, which is then more and more pronounced (14). This increase in immunotolerance leads to tumor growth, parenchyma invasion, blood dissemination and the development of metastases. All cells of innate and adaptive immunity are actors in these different steps. Moreover, cancer cells progressively evade immunosurveillance by altering their phenotype via immunoediting $(15,16)$. The immune-edited tumors display properties of cells that have undergone epithelialmesenchymal transition (EMT) (17). Taken together, a progressive cellular imbalance between anti- and pro-tumor effects in the TME leads inexorably to cancer development.

\section{Innate immunity}

Cells in the innate immunity compartment include myeloid cells [neutrophils, mastocytes, eosinophils, and myeloid-derived suppressive cells (MDSC)], macrophages and natural killer (NK) cells. Briefly, neutrophils are the most abundant myeloid cells. It is now known that these cells can survive 4 or 5 days in the blood stream and can live for several weeks in tissues (18). The neutrophils in lung carcinomas can polarize into $\mathrm{N} 1$ or $\mathrm{N} 2$ functional phenotypes (Figure 1A). N1-neutrophils express anti-tumor cytokines (such as TNF- $\alpha$ and IL-12). N2-neutrophils express pro-angiogenic (VEGF) and immunosuppressive (TGF- $\beta 1$ ) cytokines (19). As for neutrophils, macrophages have two functional phenotypes (Figure 1B). M1macrophages are induced through IFN- $\gamma$ or Tolllike receptor activation and express high levels of proinflammatory cytokines. M2-macrophages are induced by 
IL-13 and IL-4 and express different cytokines/chemokines such as PDGF, TGF- $\beta 1$, M-CSF, MCP-1 and IL-8, which are implicated in fibroblast activation and in increased angiogenesis and VEGF expression (20). Moreover, NK cells are cytotoxic lymphocytes (21). They do not need any priming to exert an anti-tumor effect (21-23). NK cells are activated by molecules (MICA and MICB) expressed by stressed tumor cells and are recognized by NK cell receptors (NKG2D) (21). Moreover tumors cells that express major histocompatibility complex class I molecules at a lower level than healthy cells can be the target of NK cells (21). Dendritic cells (DCs) are professional antigen presenting cells that sample the microenvironment and can provide different antigens and also co-stimulatory signals to the different population of cells of the adaptative immune system (24). Different populations of DCs exist in humans and could be classified into two main groups, namely, plasmacytoid DCs (pDCs) and "classical" or "myeloid" DCs. Moreover, classical or myeloid DCs have been subdivided into two subsets on the basis of their expression of CD141 and CD1c (25). DCs are critical and represent mandatory bridge acting between innate and adaptive immunity (26). These cells process phagocytosed tumor cell proteins such as peptides of CD8+ T lymphocytes to initiate reactions of adaptive immunity $(24,26,27)$. Some molecules such as CD47, a widely expressed cellular receptor well known for its immunoregulatory functions can modulate cellular phagocytosis by activation of dendritic cells and macrophages (28), It is noteworthy that T cell priming can occur not only in lymph nodes and secondary lymphoid structures, but also within lymphoid aggregates, which are located around some lung cancers, they are called TLSs $(29,30)$. All the cells of innate immunity play a pivotal role in the initiation and activation of the different cells composing the adaptive immune system.

\section{Adaptive immunity}

The cells of the adaptive immunity include $\mathrm{T}(\mathrm{CD} 8+$ and CD4+) and B cells. CD8+ T lymphocytes can destroy lung carcinoma cells through the presence of neoantigens on their membrane and through HLA class I molecules. CD4+ T lymphocytes play many roles since they recruit neutrophils and activate DC to increase CD8+ T lymphocyte priming. It is noteworthy that CD4+ T lymphocytes have different functional orientations and become Th1, Th2, Th17 and regulatory $\mathrm{T}$ cells (Tregs). Tregs suppress the effector cell responses through the production of IL-10, TGF- $\beta$ and IL-35 and the inhibition of DC maturation. Finally B cells can act as antigen-presenting cells and thus can prime $\mathrm{T}$ lymphocytes. Moreover, antibodies secreted by these cells can opsonize cancer cells, activate the complement cascade and thus induce NK cell-mediated tumor death.

\section{The switch in the battle of tumor immunity: from immune-destruction to immunotolerance}

It is well known that tumor-infiltrating ICs of lung carcinoma are plastic. These cells can have pro- or anticancer functional phenotypes. Neutrophils are activated by some damage-associated molecular patterns derived from apoptotic or necrotic cancer cells and thus can first acquire an anti-tumor (N1-neutrophils) and then a pro-tumor (N2-neutrophils) activity. In parallel M1 macrophages begin to promote cancer cell elimination in association with the activation of cells of adaptive immunity, then M2 macrophages increased in number, in particular through TGF- $\beta$ which induces an immunosuppressive effect. The anti-tumor effect is initially mediated by intra-cancer NK cells, since these cells destroy cancer cells expressing MICA and MICB with downregulated HLA-class I molecules. The activity of NK cells is mainly regulated by type I interferons and NK cells can regulate both the innate and adaptative immune responses through the secretion of many cytokines, such as the IFN- $\gamma(31)$. However the poor infiltration of NK cells into solid tumors changes in activating/inhibitory signaling and the TME decrease the NK-mediated killing of malignant cells. It is noteworthy that NK cells can inactivated by different cells such as some subpopulation of T lymphocytes (Tregs) and MDSCs but also by soluble mediators such as adenosine $(32,33)$ Loss of the ability to present neoantigens through human leukocyte antigen loss facilitates immune evasion and M2 macrophage subtype and CD8 infiltration (16,34,35). An anti-tumor efficiency needs to be associated with mature DCs, since these mature cells present the tumor peptides to CD8+ lymphocytes (27). It is then pivotal for an anti-tumor effect that the cytokine milieu include specific molecules such as TNF- $\alpha$, IL-2, IL-12 and INF- $\gamma(36,37)$. However, immune tolerance progressively develops in association with tumor cell proliferation due to the effect of renewed cytokine production including that of VEGF, IL-10, IL-35, PDGF and TGF- $\beta 1(38,39)$. Progressive accumulation of Tregs, MDSC, immature DCs, N2 neutrophils and M2- 
macrophages leads to tumor growth and cancer cell invasion and dissemination $(20,38)$. Moreover, in this context it is well known that some membrane expressed molecules (such as PD-L1) inhibit the killer function of infiltrating lymphocytes through a PD1-PD-L1 interaction, which is the biological basis of the current ICIs used in treatment of lung cancer.

\section{Intra-tumoral myeloid cell populations: a role in lung cancer immunotherapy}

The role of different populations of macrophages associated with tumors has been extensively studies these last few years $(40,41)$. Conversely, for many years myeloid cell populations have essentially been studied and characterized for their role in fighting pathogens and exogenous bodies. So the involvement of neutrophils in tumor development and progression has been underestimated until recently, since neutrophils were regarded in particular as differentiated cells with a low level of transcriptional plasticity. Thus, in the absence of stimuli neutrophils undergo apoptosis or are phagocytosed by macrophages, preventing release of cytotoxic enzymes. More recently, it was demonstrated in several studies that the different populations of myeloid cells are in fact strongly involved in carcinogenesis, tumor growth, dissemination and metastatic onset (42-45). It appears that neutrophils are the most abundant intratumor ICs type in lung tumors, accounting for around $20 \%$ of the ICs infiltrates according to the histological subtype (46). It is noteworthy that neutrophils are globally more abundant in squamous cell lung carcinoma than in lung adenocarcinoma (46). Moreover the number of neutrophils is variable in a same histological subtype, but a direct contact between tumor cells and myeloid cells can be observed, sometimes without any morphological alteration of the cancer cells (Figure 2). Globally these cells can release oxygen- and nitrogen-free radicals inducing genetic instability through the promotion of DNA point mutations in tumor cells. Different studies have revealed distinct phenotypes of neutrophils, macrophages and monocytes in tumor patients (47). Using next generation sequencing, including RNA sequencing, a number of neutrophil subtypes with high transcriptional plasticity have been reported (48). These distinct phenotypes could be the result of the reprogramming of the tissue microenvironment or of the development of specific progenitor cells. So, it was demonstrated that neutrophils in lung cancers express different phenotypes that play both pro- and anti-tumor roles (49). Anti-tumor neutrophils (type 1) are usually observed during early stage lung cancers and stimulate INF- $\gamma$ production and T cell infiltration. During tumor progression a neutrophil-rich infiltrate correlates with a decrease in CD8+ and CD4+ cells and a lower overall survival of patients with NSCLC (50). Kargl and colleagues deciphered the myeloid lineage cellular population in NSCLC and their association with lymphocyte populations (50). For this purpose they used different approaches and a multiparametric flow data set, a Nanostring Immune Profiler gene expression panel and a novel multiplex immunohistochemistry (IHC) panel. Interestingly the multiplex-IHC panel was designed to address potential roles of M-MDSC, neutrophils, macrophages and monocytes in the mediation of lymphocytes (50). This study highlighted the ratio of $\mathrm{CD} 8+\mathrm{T}$ to neutrophils within tumors optimally distinguishing between active and myeloid cases (50).

Among the myeloid cell populations observed in lung carcinomas, the MDCSs subset is an important highly immunosuppressive population (51). These MDSCs express CD45 and CD33, but not CD14, CD68 and CD66b. Recently the expression of checkpoint molecules on neutrophils and MDSCs was demonstrated by different studies and interest was shown using mice models in inducing targeted deletion of PD-1 or of PD-L1 in myeloid cells to bring about an anti-tumor immune reaction $(52,53)$. Notably, it was demonstrated a high expression of PD-L1 protein on human granulocytic MDSCs upon co-culture with $\mathrm{T}$ cells (52). Functional studies further demonstrated that antibody blocking PD-L1 impaired the MDSCmediated suppression of T-cell proliferation $(52,54,55)$. Interestingly, hypoxia, one of the hallmarks of cancer, plays a critical role in anti-cancer immune responses by not only reducing cytolytic and migratory activity of effector cells such as CD8+ T and NK cells, but also by supporting immunosuppressive cells, notably the MDSCs $(54,55)$.

Several mechanisms are used by lung cancer cells undergoing EMT to interact with ICs, notably myeloid populations, allowing angiogenesis, tumor growth and dissemination. Thus, tumor cells that have undergone EMT can modify the TME and affect different IC populations with an increase in the number of immunosuppressive cells such as MDSCs, tumor-associated macrophages with a M2 phenotype and Treg lymphocytes. These cells release immunosuppressive molecules (IL-10, TGF- $\beta$, TNF- $\alpha$ ), which inhibit cytotoxic cells, notably CD8+ T lymphocytes and NK cells. Production of some cytokines, such as TGF- $\beta$ induces EMT and also impairs differentiation of 

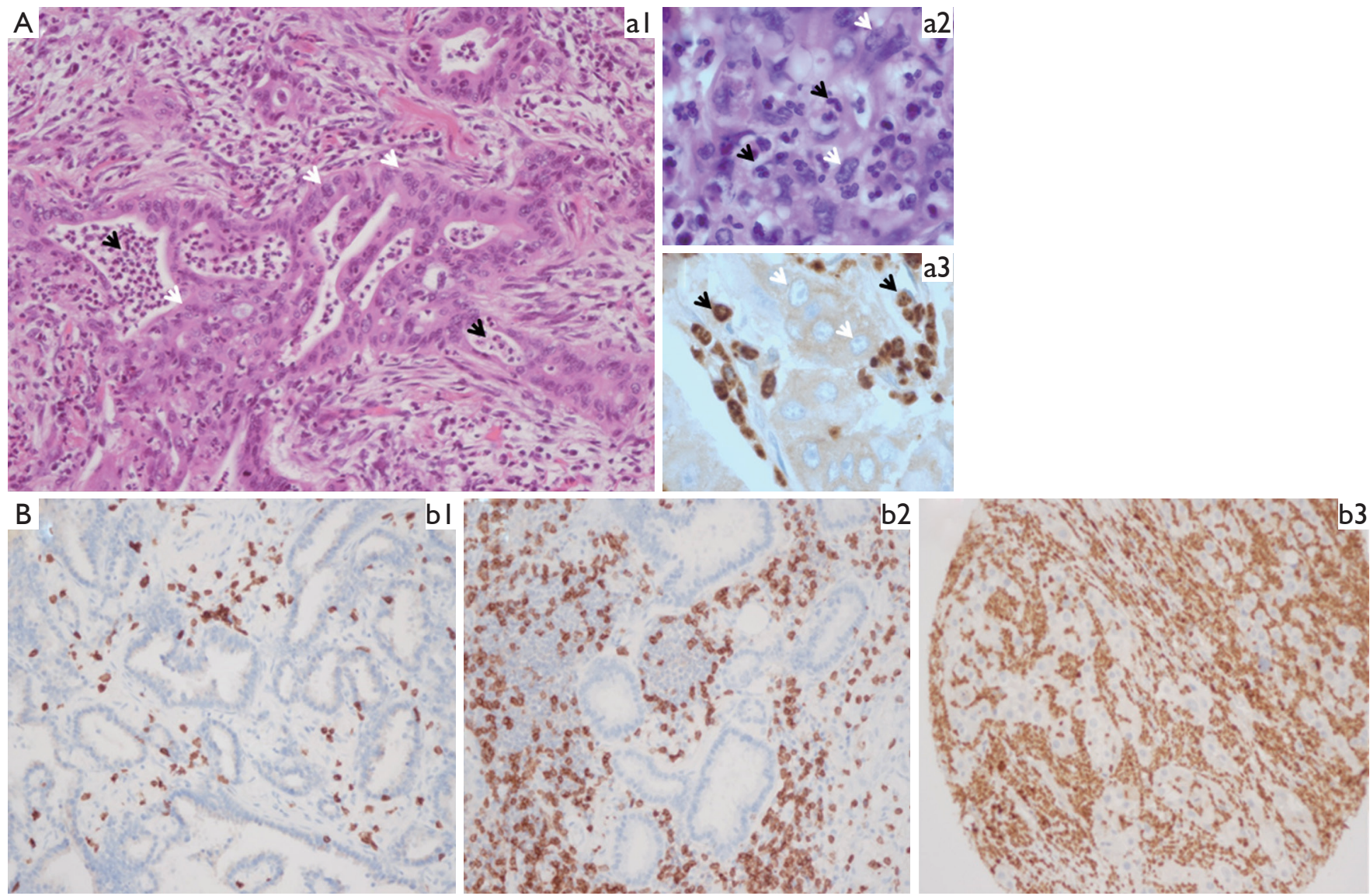

Figure 2 Myeloid cells infiltration in tissue sections of lung carcinoma. (A) Interaction and cell-cell contact between lung cancer tumor cells (white arrows) and neutrophils (black arrows). (a1) Hematoxylin-eosin, original magnification $\times 200$, (a2) hematoxylin-eosin, original magnification $\times 630$, (a3) immunohistochemistry with an anti-myeloperoxidase antibody, immunoperoxidase, original magnification $\times 800$. (B) Different lung adenocarcinoma showing low (b1), moderate (b2) and high (b3) number of myeloid cells (b1-b3: anti-myeloperoxidase antibody; immunoperoxidase, original magnification $\times 100)$.

many innate and adaptive ICs of the TME in lung cancer. Interestingly, in vitro studies demonstrated that TGF- $\beta$ could also downregulate the MHC class I proteins on lung cancer cells (56). EMT has been found to be associate with an increased level of different ICIs, including PD-1, PDL1 and PD-L2, OX40, CD137, TIM3, LAG3 and CTLA-4 (57-59). Finally, substantial interplay exists between myeloid populations and microRNAs in the TME. More notably some miRNAs can be produced by both tumor cells and/or myeloid cells and can modify tumor development and dissemination, such as miR223 produce mainly by neutrophils or miR21 and miR29 that are produced by macrophages (60-62). Interestingly, these miRNAs, which are mainly present in extra vesicles, play an important role in resistance to immunotherapy (63).

\section{The place of TLSs in the concept of immunotherapy}

Lung parenchyma can develop TLSs in association with different diseases including non-tumor and tumor processes. TLSs correspond to lymphoid structures that develop in non-lymphoid tissues $(29,30)$. Development is linked to long-term exposure to chronic inflammatory signals. Moreover, TLSs are described in the invasive margin but also in the stroma of some lung tumors. Some lung cancer patients can present with many TLSs in their tumors while others do not show any of these structures. As described for lymphoid tissues, TLSs are composed of different populations of ICs, B cell follicles with germinal centers, surrounded by plasma cells and T cell-rich zones 
with mature DCs. Moreover these TLSs showed a high number of endothelial venules. The composition can be analyzed using a M-multiplex IHC approach (64). As in lymphoid tissues, local presentation of tumor antigens to $\mathrm{T}$ lymphocytes by DCs, differentiation of B and T cells, as well as generation of memory B cells, antibody production by plasma cells, and production of $\mathrm{T}$ cytotoxic and $\mathrm{T}$ helper populations, can be observed in TLSs. It is noteworthy that there is a strong correlation between the presence of a high number of CD4+ $\mathrm{T}$ and $\mathrm{CD} 8+\mathrm{T}$ lymphocytes in lung carcinomas and the presence of TLSs. Moreover, the presence of a high number of CD8+PD1+ T lymphocytes in TLSs before immunotherapy was found to be predictive of response to this treatment (65). A favorable impact of the TLS number detected in tissue sections on prognosis of lung cancer has been reported (66). This prognostic factor was totally independent of the pTNM staging (66). When deciphering the different cell populations of TLS high tumors, a high level of CD38+ and CD69+ activated T cells and of CD8+ T cells was demonstrated. Moreover, a high number of genes characteristic of $\mathrm{T}$ cell activation, $\mathrm{T}$ cell cytotoxicity and $\mathrm{T}$ cell chemotaxis was described (66).

\section{ATP and P2R: potential new actors in lung cancer immunotherapy}

One of the most potent immunosuppressive factors in solid tumors, notably in lung cancer, is adenosine, which is produced in the tumor stroma when extracellular ATP is degraded (67-69). Adenosine can impair antitumor activity, through the decrease of protective ICs (such as $\mathrm{T}$ cells, NK cells and DCs), by enhancing the suppressive capacity of Tregs and MDSCs $(67,68)$. So adenosine confers potent immunosuppressive as well direct tumorpromoting actions in the lung TME. ATP acts at P2Rs expressed on both tumor and host cells (70,71) (Figure 3). Depending on the extracellular ATP level, the P2Rs subtype and the target cell type (tumor or healthy cells) stimulation of $\mathrm{P} 2$ receptors has different effects. P2Rs are plasma membrane receptors, which are stimulated by pyrimidine nucleotides or extracellular purine. The P2R family is made up of eight P2Y and seven P2X members (with the receptor family comprising adenosine receptors named $\mathrm{P} 1$ purinergic receptors). The affinity for ATP of these different receptors varies strongly according the ATP concentration. Among the $\mathrm{P} 2$ receptors, the $\mathrm{P} 2 \mathrm{X} 7 \mathrm{R}$ subtype appears to be a major player not only in some inflammatory diseases but also in host-tumor cell interactions (72-75). Interestingly P2X7R is expressed on tumor cells but also on some intra-tumoral ICs including MDSC, NK cells and lymphocytes. ATP is released into the TME and can modulate the interaction between intra-tumor ICs and the cells of the carcinoma. Cell injury, dying cells and hypoxia are strong stimuli for ATP release. In solid tumors, ATP and ADP are degraded by membrane-expressed ectonucleotidases such as CD39. Similarly, AMP is also degraded by CD73 membrane-bound ectonucleotidase in solid tumors. It is noteworthy that CD39 is strongly expressed by Tregs, T and B lymphocytes, DCs, tumor-infiltrating TH17 lymphocytes and M2 macrophages. Moreover, CD73 and CD39 are overexpressed in response to TGF- $\beta$. Globally low ATP levels promote cancer cell proliferation and immunosuppression while high levels of ATP activate DCs, promote tumor antigen presentation and are associated with an anti-tumor effect. However, ATP can also induce immunosuppression by causing adenosine accumulation. ATP can selectively increase the number of immunosuppressive cells in the TME by increasing chemotaxis of Treg lymphocytes. Moreover, ATP can contribute to MDSC immunosuppressive functions as a substrate for the generation of 5'AMP. In this regard, ATP stimulation of P2X7R on MDSCs drives arginase accumulation, but also TGF- $\beta$ and IL-10 release and strongly contributes to the onset of elaboration of an immunosuppressive TME. In parallel, DCs express a large number of P2Rs and migration of DCs can be increased by extracellular nucleotides. Interestingly, ATP promotes matrix metallopeptidase 9 and cathepsin production, and inhibits tissue inhibitor of metalloproteinases 1 release from monocytes. Thus, this process can induce degradation of the extracellular matrix to facilitate lung carcinoma cell invasion and ICs infiltration. However, cancer-associated activity can be stimulated by adenosine via A2bR. Finally, release of VEGF from tumor associated macrophages and cancer cells can be promoted by adenosine acting on A2bR but also by ATP acting on P2X7R.

Some studies report expression of CD39 on TILs, in particular in CD8+ TILs in NSCLC $(76,77)$. A recent study showed that CD39 and PD-1 can be co-expressed in tumor-infiltrating ICs in NSCLC (78). Thus, PD-1 and adenosine signaling pathways for tumor immune-escape and immunosuppression can occur simultaneously. Expression of these markers was found to be higher in both CD4+ and $\mathrm{CD} 8+\mathrm{T}$ cells isolated from tumor tissue than in these cells isolated from adjacent non tumor tissue (78). 

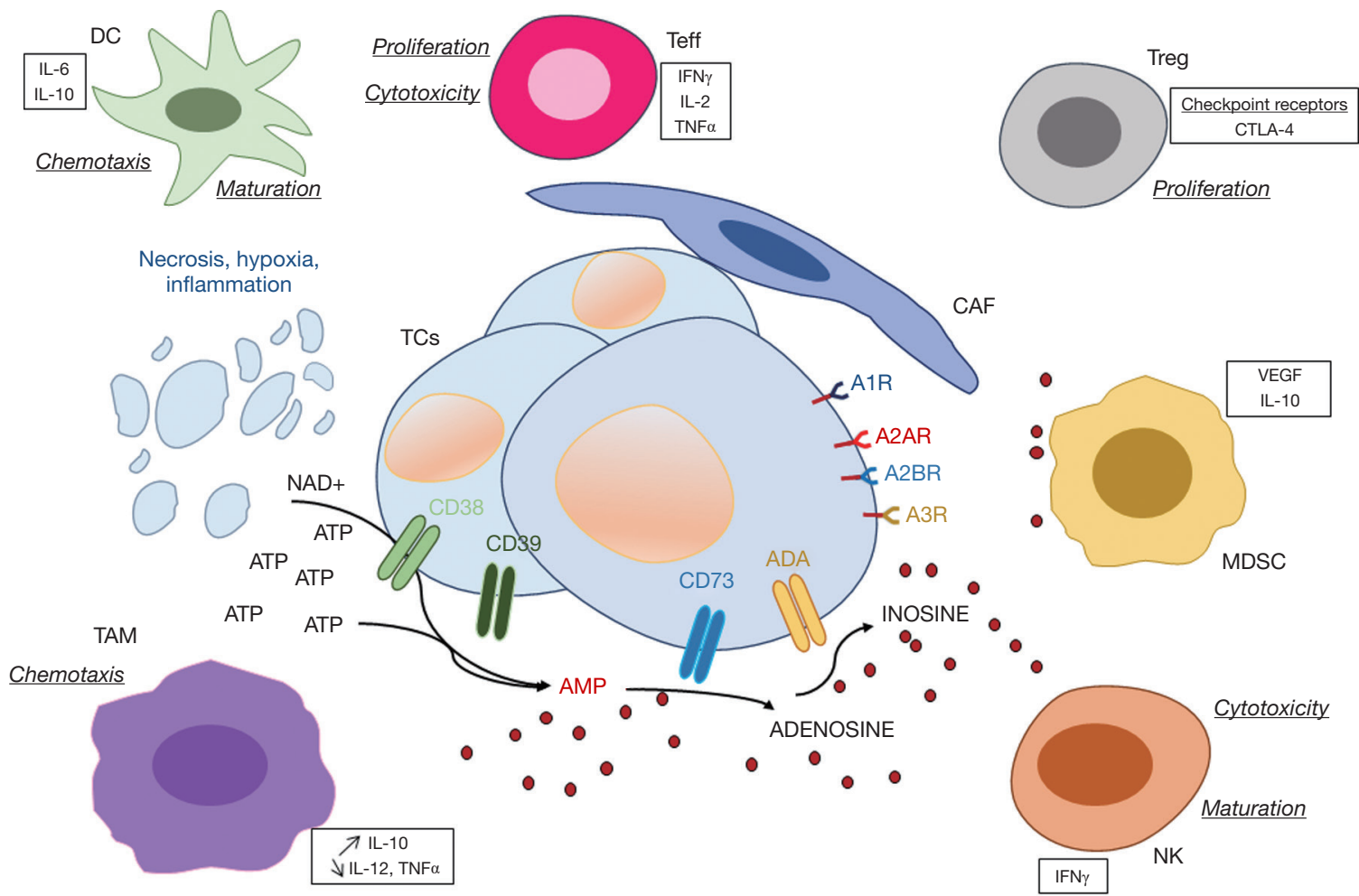

Figure 3 Impact of an increase level of adenosine in the different cells of the tumor microenvironment. Injured tissue (and its consequences such as necrosis, hypoxia and/or inflammation) increases the level of ATP and NAD+ in the interstitial compartment. ATP and NAD+ fuel catabolic adenosine-generating pathways which can be mediated by CD39 and CD73, but also other ecto-nucleotidases including CD38. Adenosine can be eliminated via its conversion to inosine by ADA receptor. Four adenosine receptors (AR) all coupled to G-proteins have identified: AIR, A2AR, A2BR and A3R. AI, A2A and A3 are high affinity adenosine receptor and A2B is low affinity adenosine receptor. Adenosine induces different functions in the immune subsets and the secretion of many cytokines allowing tumor progression. DC, dendritic cell; TAM, tumor-associated macrophage; Treg, T regulator lymphocyte; Teff, T effector lymphocyte; CAF, carcinoma cell-associated fibroblast; MDSC, myeloid-derived suppressor cell; NK, natural killer cell.

\section{The "liquid microenvironment": a new space for lung cancer immunotherapy development}

The onset of lung metastases is mainly due to the hematogenous dissemination of tumor cells, called circulating tumor cells (CTCs), arising from the primary cancer site (79). However, the majority of carcinoma cells once present and circulating in the blood do not survive and are not able to create secondary metastases. So, most of these CTCs die due to apoptotic phenomena, called anoikis. However, a subpopulation of CTCs, which form in clusters called tumor microemboli, resist the blood flow, the pressure and turbulence, and so survive and develop metastases. So, it is pivotal to decipher the molecular mechanisms associated with the survival or death of CTCs. One of the main influences on the behavior CTCs is driven by their interaction with circulating hematological cells, in particular, neutrophils, MDSC, macrophages/monocytes, NK, and platelets (80-82). These interactions are based on cell-cell contact as well as through the action of some soluble factors secreted by the CTCs and/or the nontumoral circulating cells, as well as through some microparticles, including exosomes (81). As for tumor tissue, the ICs in blood can have an anti- or pro-tumor action, and can also combine together their effects (82-84). Briefly, circulating neutrophils and macrophages can have an $\mathrm{N} 1$ or M1, or an N2 or M2 phenotype, respectively $(80,81)$. More notably, these cells can produce some neutrophil extracellular traps (NETs) and actively participate in CTCs survival through their action on the cell cycle, which increases the resistance to apoptosis, and is involved in the 
onset of metastases (84). After activation and aggregation circulating platelets associated with NETs can favor the adherence of CTCs onto endothelial cells and then their extravasation, steps arising before metastatic development $(80,81)$. It is noteworthy that neutrophils are involved in all the steps associated with cancer dissemination, notably at an early stage when they support primary cancer cell entry into the blood stream, through the secretion of proteases, elastases and metalloproteases that destroy the vasculature barrier $(80,81)$. The neutrophils then escort the CTCs into the blood stream creating a protective niche, and finally allowing them to cross the vascular barrier $(80,81)$. Moreover, the neutrophils prepare the site for CTC implantation in distant parenchyma from different organs. NK cells are strongly involved in eliminating CTCs, but their activity can be inhibited by some soluble factors, such as TGF- $\beta$ secreted by M2 macrophages $(80,81)$. Finally MDSC can act by inhibiting the cytotoxic effect of circulating CD8+ lymphocytes (80).

\section{Current and future tools to study the interactions between immune and cancer cells}

Many tools and approaches have been developed to analyze the TME in lung cancer in order to quantifying and characterizing the IC populations. Some of these tools will be used very soon in daily practice to better evaluate the prognosis but also to help increase the predictive value of response or resistance to lung cancer therapy, notably immunotherapy.

\section{IHC and immunofluorescence (IF)}

IHC and IF are methods used worldwide in pathology laboratories. In routine pathology, IHC is easy to perform, cost effective, and the results are obtained rapidly. Moreover, one of the advantages of these methods is to identify the signal at the architectural level and to localize the detected protein in the different cell compartments, i.e., in the nucleus, the cytoplasm and/or the cell membrane. Recently, multiplex IHC or multiplex IF have been developed to detect and localize in a single tissue section at the same time several antigens $(85,86)$. Depending on the technology (chromogenic versus fluorescence approaches), the number of antibodies which can be used is variable from two to ten, sometimes more $(85,87,88)$. The different IC populations present in the lung cancer tissue section can be detected and quantified (89). One of the advantages of these methods is the possibility of studying in detail the spatial topography of these complex cellular infiltrates, of being able to assess the co-localization of the different detected molecules, and according to the method, of evaluating the distance between the antigens. Currently the great majority of multiplex IHC/IF tools have been developed for translational research programs, but some new approaches should be applied soon in routine clinical practice for detection of both ICs, but also of well-defined predictive biomarkers (90).

\section{Flow and mass cytometry}

Cytometry is certainly one of the best methods to study the functionality of ICs in a lung carcinoma. However, analysis by flow cytometry requires the dissociation of the tumor tissue to stain a single-cell suspension since each cell marker is read with a laser $(91,92)$. For example, this technique can evaluate the profile of tumor-associated lymphocytes to point to the prognosis as well as the patient's response to therapy. Each IC population of interest can be analyzed for multi-parametric expression of many markers (92). Moreover, the data of a well-defined single cell population of IC can quantify millions of cells in a short turnaround time (91). In addition, different populations of millions ICs can be analyzed at the same time. This method is very useful for the study of the phenotype of rare IC population such as DCs or MDSCs. In addition to flow cytometry mass cytometry allows evaluation of several dozen markers all at the same time. For this method, the cells are labeled with metal-tagged antibodies, which are then quantified by time of flight mass spectrometry. For example, using this approach it was possible to study in detail IC populations in lung carcinoma, and by coupling this method with single cell RNA it was possible to evaluate and quantify the NK, myeloid and T cells compartment in early lung adenocarcinomas $(49,93)$.

\section{Gene expression data}

Different methods have been created to analyze the transcriptomic data of lung tumors to decipher the composition of the cell populations of the TME. These methods are based on Gene Set Enrichment Analysis and can also quantify the different cell populations with the TIminer and xCell methods. The MCP-counter was more recently developed and is based on an approach using gene signatures. Signatures are available for eight immune 
populations, as well as fibroblasts and the endothelium. Other methods including CIBERSORT and TIMER, have developed a deconvolution framework. Different methods can perform complete deconvolution that simultaneously estimates the proportion of cell type and of their transcriptomic profiles. This is possible for quite low numbers of cell populations. Finally, other methods, such as ISOpure for example, can separate the part of the transcriptome that is related to tumor or healthy cells. Gene expression biomarkers in NSCLC, notably predefined immune gene signatures, have been recently evaluated using the NanoString PanCancer IO360 panel code (Nanostring, CA USA) (94). It is noteworthy that the tumor inflammatory signature was predictive of the benefit of ICIs in a cohort of lung cancer patients (94). One of the main disadvantages of transcriptomic methods is the loss of knowledge and evaluation concerning the tumor architecture and the spatial data required to localize identified cell types in the tumor, notably the ICs populations. However, some studies use barcodes associated with different regions of the analyzed tissue before doing RNA sequencing, allowing a spatial transcriptomic method (95).

Analyzing ICs at the single-cell scale by using single-cell RNA sequencing technologies can allow the assessment of the cell phenotype as well as its functional status (96-98). In this context this approach can identify the $\mathrm{T}$ cell receptor repertoire (97). Moreover, this technology allows detail study of rare ICs populations, since it is very hard to segregate from background these populations in transcriptomics.

\section{New potential therapeutic strategies targeting the TME}

Anti-PD1/PD-L1 molecules administered alone or in combination with chemotherapy allow a significant higher overall survival in advanced and metastatic NSCLC (99-103). However, this dramatic result is observed in only a minority of lung cancer patients. So there is an urgent need to discover new therapeutic molecules to increase the number of patients who benefit from immunotherapy. Development of molecules targeting cells of the TME are ongoing. Studies use preclinical animal and cellular models and molecules are tested in an increasing number of clinical trials (104-110). Here we describe briefly some of these therapeutic options, focusing on the topics described above.

Different therapeutic molecules targeting innate immunity, notably the myeloid populations and/or their secreted molecules are currently being tested in clinical trials (111,112). Macrophage-centered therapeutic development focuses on activating the macrophage anticancer activity or on inhibiting their recruitment $(113,114)$. Many strategies focus on targeting the macrophages, notably the M2 macrophage population $(113,114)$. These strategies aim to inhibit pro-tumor macrophage recruitment and localization but also to activate M1 macrophage populations. A number of different antibodies and molecules have been or are being tested on cancer patients, including selective antibodies to CCL2, CCR2, CCR5, CSF1R antagonists. Other molecules such as the retinoicacid-related orphan receptor, which is expressed by myeloid cells, is another potential macrophage target in lung cancer. Many other molecules are also being developed and/or have been evaluated, such as some bisphosphonates, trabectedin or anti-CD40 molecules. Other therapeutic strategies are ongoing as for targeted neutrophils, or MDSC populations and/or some cytokines secreted by these cells, such as the IL-8 (112,115). These include the potential for inhibition of CXC receptors like CXCR2 that are associated with the migration of neutrophils to tumor sites. Other strategies focus on the inhibition of IL-23 or IL-17 since these cytokines stimulate the expansion of neutrophils, which is mediated by G-CSF. However, it is also possible to directly target G-CSF (115). In addition, many scientific reports and clinical studies have shown promising anti-tumor effects when using NK cell-based immunotherapy $(22,23,116)$. Currently, various approaches are being used to enhance the number and function of NK cells (116). One approach uses cytokines to selectively boost both the number as well as the efficacy of anti-tumor functions of NK cells (116). Another emerging approach focuses on checkpoint inhibitors targeting the NK cell receptor $(22,23)$.

The induction of TLS formation and the increase in their number due to different pharmacological approaches may be useful to increase the sensitivity of the "cold tumors" to ICIs, notably when used in association with ICIs, vaccines and/or intra-tumor agents. However, in some "hot tumors" with fibrotic stroma, angiogenesis, and chronic inflammation, TLS formation and the response to ICIs can also be favored by some anti-immunosuppressive and/or anti-angiogenic molecules. The induction of TLSs in lung carcinoma can facilitate the lymphocyte afflux and tumor control. In this context, TLS induction may be used for treatment of patients with some tumors with a low level of lymphocyte infiltration, notably a low level of CD8+ lymphocytes. The promotion of TLSs may be obtained 
using different chemokines, synthetic molecules, antigenpresenting cells, antibodies and/or cytokines. In addition, TLSs can represent a target of choice to produce and test efficient anti-tumor vaccine therapies since they can generate an efficient adaptive anti-tumor signal (117).

Purinergic signaling may be a new target for lung cancer therapy. Targets would include the different P2 receptors as well as extracellular ATP. The in vivo injection of ATP has been explored as an anti-cancer therapy in mice models, but some conflicting results were obtained. Notably administration of $500 \mathrm{mg}$ per kilogram of ATP intra-peritoneal was associated with tumor regression via activation of $\mathrm{P} 2 \mathrm{X} 7 \mathrm{R}$ (118). However, injection of a high dose of ATP can be counterproductive since it leads to a large quantity of adenosine and an increased immunosuppressive effect. Some projects are focusing on the inhibition of ATP release into the TME. Suitable targets include pannexin 1 and P2X7R. Alternatively, it may be possible to administrate recombinant soluble CD39. Preclinical studies in several tumor models have shown that targeting $\mathrm{P} 2 \mathrm{X} 7 \mathrm{R}$ is potentially a very effective anti-cancer treatment, and many pharmaceutical companies have now developed potent and selective small molecule inhibitors of P2X7R.

Improvement in knowledge concerning the pathophysiology of the CTC-blood cell interactions opens new avenues to therapeutic development. For example, it has been shown that P2Y12R can play a pivotal role in platelet activation and clot formation. In preclinical models it has been demonstrated that platelet P2Y12R contributes to both tumor growth and dissemination. In this context, P212R-deficient mice showed reduced lung cancer cell dissemination owing to disruption in tumor-platelet interactions (119). Among the other strategies, one focuses on inhibiting NET formation by administering DNase I, which reverses the effect of some pro-tumor factors. However, these approaches are currently controversial since NETs may have anti- or pro-tumor effects (120). Many molecules are in development or in use in different clinical trials, and may soon be in used in daily practice. These molecules have been developed based on improved knowledge into the function of the immune system in association with lung cancer cell activity.

\section{Perspectives and conclusion}

The composition and density of ICs in the TME of lung carcinomas is variable from a tumor to another one, but has to be considered with the number and type of associated genomic alterations $(121,122)$. Notably, KRAS mutations influence and modify the composition of the different intra tumoral ICs $(121,123,124)$. A number of reports have shown that KRAS mutated lung cancers demonstrated an increase in PD-L1 expression and secretion of pro-tumoral cytokines (such as IL-8, IL-17, and IL-22) and have different IC populations depending on the KRAS mutation subtypes $(121,123,124)$. One of the most recent exciting therapeutic molecules, a G12C-specific inhibitor, targets KRASG12C mutant lung tumors, and opens new opportunities to treat lung adenocarcinoma patients using this inhibitor alone or in association with an ICI (125-128).

Many developments and approaches are currently being studied to better analyze and quantify the ICs in the lung cancer TME. For example, approaches using histologybased digital-staining, a deep learning-based computation model, or different programs using artificial intelligence tools will certainly be helpful in the near future to better evaluate the composition of ICs in lung cancer, as well as their prognostic and predictive value, in particular in patients treated with ICIs $(129,130)$.

\section{Acknowledgments}

The author wishes to thank Ms Christelle Bonnetaud, Ms Christiane Brahimi-Horn, the Ligue Départementale 06, the Conseil Départemental 06 des Alpes Maritimes and the Cancéropôle PACA for their support.

Funding: None.

\section{Footnote}

Provenance and Peer Review: This article was commissioned by the Guest Editor (Helmut H. Popper) for the series "New Developments in Lung Cancer Diagnosis and Pathological Patient Management Strategies" published in Translational Lung Cancer Research. The article was sent for external peer review organized by the Guest Editor and the editorial office.

Conflicts of Interest: The author has completed the ICMJE uniform disclosure form (available at http://dx.doi. org/10.21037/tlcr-20-178). The series "New Developments in Lung Cancer Diagnosis and Pathological Patient Management Strategies" was commissioned by the editorial office without any funding or sponsorship. PH serves as an unpaid editorial board member of Translational Lung Cancer 
Research from Feb 2019 to Feb 2021.

Ethical Statement: The author is accountable for all aspects of the work in ensuring that questions related to the accuracy or integrity of any part of the work are appropriately investigated and resolved.

Open Access Statement: This is an Open Access article distributed in accordance with the Creative Commons Attribution-NonCommercial-NoDerivs 4.0 International License (CC BY-NC-ND 4.0), which permits the noncommercial replication and distribution of the article with the strict proviso that no changes or edits are made and the original work is properly cited (including links to both the formal publication through the relevant DOI and the license). See: https://creativecommons.org/licenses/by-nc-nd/4.0/.

\section{References}

1. Fridman WH, Zitvogel L, Sautès-Fridman C, et al. The immune contexture in cancer prognosis and treatment. Nat Rev Clin Oncol 2017;14:717-34.

2. Remark R, Becker C, Gomez JE, et al. The non-small cell lung cancer immune contexture. A major determinant of tumor characteristics and patient outcome. Am J Respir Crit Care Med 2015;191:377-90.

3. Taube JM, Galon J, Sholl LM, et al. Implications of the tumor immune microenvironment for staging and therapeutics. Mod Pathol 2018;31:214-34.

4. da Silva JL, Dos Santos ALS, Nunes NCC, et al. Cancer immunotherapy: the art of targeting the tumor immune microenvironment. Cancer Chemother Pharmacol 2019;84:227-40.

5. Frankel T, Lanfranca MP, Zou W. The Role of Tumor Microenvironment in Cancer Immunotherapy. Adv Exp Med Biol 2017;1036:51-64.

6. Galon J, Bruni D. Approaches to treat immune hot, altered and cold tumours with combination immunotherapies. Nat Rev Drug Discov 2019;18:197-218.

7. Hui L, Chen Y. Tumor microenvironment: Sanctuary of the devil. Cancer Lett 2015;368:7-13.

8. Remon J, Passiglia F, Ahn MJ, et al. Immune Checkpoint Inhibitors in Thoracic Malignancies: Review of the Existing Evidence by an IASLC Expert Panel and Recommendations. J Thorac Oncol 2020. doi:10.1016/ j.jtho.2020.03.006.

9. Wilky BA. Immune checkpoint inhibitors: The linchpins of modern immunotherapy. Immunol Rev 2019;290:6-23.
10. Xia L, Liu Y, Wang Y. PD-1/PD-L1 Blockade Therapy in Advanced Non-Small-Cell Lung Cancer: Current Status and Future Directions. Oncologist 2019;24:S31-S41.

11. Abril-Rodriguez G, Ribas A. SnapShot: Immune Checkpoint Inhibitors. Cancer Cell 2017;31:848-848.e1.

12. Hegde PS, Chen DS. Top 10 Challenges in Cancer Immunotherapy. Immunity 2020;52:17-35.

13. Kawakami Y, Ohta S, Sayem MA, et al. Immune-resistant mechanisms in cancer immunotherapy. Int J Clin Oncol 2020. doi: 10.1007/s10147-019-01611-x.

14. Finn OJ. A Believer's Overview of Cancer Immunosurveillance and Immunotherapy. J Immunol 2018;200:385-91.

15. O'Donnell JS, Teng MWL, Smyth MJ. Cancer immunoediting and resistance to $T$ cell-based immunotherapy. Nat Rev Clin Oncol 2019;16:151-67.

16. Rosenthal R, Cadieux EL, Salgado R, et al. Neoantigendirected immune escape in lung cancer evolution. Nature 2019;567:479-85.

17. Soundararajan R, Fradette JJ, Konen JM, et al. Targeting the Interplay between Epithelial-to-MesenchymalTransition and the Immune System for Effective Immunotherapy. Cancers (Basel) 2019;11:714.

18. Kolaczkowska E, Kubes P. Neutrophil recruitment and function in health and inflammation. Nat Rev Immunol 2013;13:159-75.

19. Tecchio C, Micheletti A, Cassatella MA. Neutrophilderived cytokines: facts beyond expression. Front Immunol 2014;5:508.

20. Goswami KK, Ghosh T, Ghosh S, et al. Tumor promoting role of anti-tumor macrophages in tumor microenvironment. Cell Immunol 2017;316:1-10.

21. Chiossone L, Dumas PY, Vienne M, et al. Natural killer cells and other innate lymphoid cells in cancer. Nat Rev Immunol 2018;18:671-88.

22. Burugu S, Dancsok AR, Nielsen TO. Emerging targets in cancer immunotherapy. Semin Cancer Biol 2018;52:39-52.

23. Choucair K, Duff JR, Cassidy CS, et al. Natural killer cells: a review of biology, therapeutic potential and challenges in treatment of solid tumors. Future Oncol 2019;15:3053-69.

24. Guilliams M, Ginhoux F, Jakubzick C, et al. Dendritic cells, monocytes and macrophages: a unified nomenclature based on ontogeny. Nat Rev Immunol 2014;14:571-8.

25. Summers KL, Hock BD, McKenzie JL, et al. Phenotypic characterization of five dendritic cell subsets in human tonsils. Am J Pathol 2001;159:285-95.

26. Lee YS, Radford KJ. The role of dendritic cells in cancer. Int Rev Cell Mol Biol 2019;348:123-78. 
27. Veglia F, Gabrilovich DI. Dendritic cells in cancer: the role revisited. Curr Opin Immunol 2017;45:43-51.

28. Liu X, Pu Y, Cron K, et al. CD47 blockade triggers T cellmediated destruction of immunogenic tumors. Nat Med 2015;21:1209-15.

29. Sautès-Fridman C, Petitprez F, Calderaro J, et al. Tertiary lymphoid structures in the era of cancer immunotherapy. Nat Rev Cancer 2019;19:307-25.

30. Teillaud JL, Dieu-Nosjean MC. Tertiary Lymphoid Structures: An Anti-tumor School for Adaptive Immune Cells and an Antibody Factory to Fight Cancer? Front Immunol 2017;8:830.

31. Sun JC, Lanier LL. NK cell development, homeostasis and function: parallels with CD8+ T cells. Nat Rev Immunol 2011;11:645-57.

32. Sek K, Mølck C, Stewart GD, et al. Targeting Adenosine Receptor Signaling in Cancer Immunotherapy. Int J Mol Sci 2018;19:3837.

33. Sordo-Bahamonde C, Vitale M, Lorenzo-Herrero S, et al. Mechanisms of Resistance to NK Cell Immunotherapy. Cancers (Basel) 2020;12:E893.

34. McGranahan N, Rosenthal R, Hiley CT, et al. AlleleSpecific HLA Loss and Immune Escape in Lung Cancer Evolution. Cell 2017;171:1259-1271.e11.

35. Perea F, Bernal M, Sánchez-Palencia A, et al. The absence of HLA class I expression in non-small cell lung cancer correlates with the tumor tissue structure and the pattern of T cell infiltration. Int J Cancer 2017;140:888-99.

36. Berraondo P, Sanmamed MF, Ochoa MC, et al. Cytokines in clinical cancer immunotherapy. Br J Cancer 2019;120:6-15.

37. Showalter A, Limaye A, Oyer JL, et al. Cytokines in immunogenic cell death: Applications for cancer immunotherapy. Cytokine 2017;97:123-32.

38. Mantovani A, Sozzani S, Locati M, et al. Macrophage polarization: tumor-associated macrophages as a paradigm for polarized M2 mononuclear phagocytes. Trends Immunol 2002;23:549-55

39. Miyazono K, Katsuno Y, Koinuma D, et al. Intracellular and extracellular TGF- $\beta$ signaling in cancer: some recent topics. Front Med 2018;12:387-411.

40. Kielbassa K, Vegna S, Ramirez C, et al. Understanding the Origin and Diversity of Macrophages to Tailor Their Targeting in Solid Cancers. Front Immunol 2019;10:2215.

41. Locati M, Curtale G, Mantovani A. Diversity, Mechanisms, and Significance of Macrophage Plasticity. Annu Rev Pathol 2020;15:123-47.

42. Ji JJ, Fan J. Discovering myeloid cell heterogeneity in the lung by means of next generation sequencing. Mil Med Res 2019;6:33.

43. Porta C, Sica A, Riboldi E. Tumor-associated myeloid cells: new understandings on their metabolic regulation and their influence in cancer immunotherapy. FEBS J 2018;285:717-33.

44. Umansky V, Adema GJ, Baran J, et al. Interactions among myeloid regulatory cells in cancer. Cancer Immunol Immunother 2019;68:645-60.

45. Wu L, Saxena S, Singh RK. Neutrophils in the Tumor Microenvironment. Adv Exp Med Biol 2020;1224:1-20.

46. Kargl J, Busch SE, Yang GH, et al. Neutrophils dominate the immune cell composition in non-small cell lung cancer. Nat Commun 2017;8:14381.

47. Zilionis R, Engblom C, Pfirschke C, et al. Single-Cell Transcriptomics of Human and Mouse Lung Cancers Reveals Conserved Myeloid Populations across Individuals and Species. Immunity 2019;50:1317-1334.e10.

48. Ng LG, Ostuni R, Hidalgo A. Heterogeneity of neutrophils. Nat Rev Immunol 2019;19:255-65.

49. Stankovic B, Bjørhovde HAK, Skarshaug R, et al. Immune Cell Composition in Human Non-small Cell Lung Cancer. Front Immunol 2019;9:3101.

50. Kargl J, Zhu X, Zhang H, et al. Neutrophil content predicts lymphocyte depletion and anti-PD1 treatment failure in NSCLC. JCI Insight 2019;4:e130850.

51. Vetsika EK, Koukos A, Kotsakis A. Myeloid-Derived Suppressor Cells: Major Figures that Shape the Immunosuppressive and Angiogenic Network in Cancer. Cells 2019;8:1647.

52. Ballbach M, Dannert A, Singh A, et al. Expression of checkpoint molecules on myeloid-derived suppressor cells. Immunol Lett 2017;192:1-6.

53. Strauss L, Mahmoud MAA, Weaver JD, et al. Targeted deletion of PD-1 in myeloid cells induces antitumor immunity. Sci Immunol 2020;5:eaay1863.

54. Dysthe M, Parihar R. Myeloid-Derived Suppressor Cells in the Tumor Microenvironment. Adv Exp Med Biol 2020;1224:117-40.

55. Yang Z, Guo J, Weng L, et al. Myeloid-derived suppressor cells-new and exciting players in lung cancer. J Hematol Oncol 2020;13:10.

56. Tripathi SC, Peters HL, Taguchi A, et al. Immunoproteasome deficiency is a feature of non-small cell lung cancer with a mesenchymal phenotype and is associated with a poor outcome. Proc Natl Acad Sci U S A 2016;113:E1555-64

57. Jiang Y, Zhan H. Communication between EMT and PD- 
L1 signaling: New insights into tumor immune evasion. Cancer Lett 2020;468:72-81.

58. Lou Y, Diao L, Cuentas ER, et al. Epithelial-Mesenchymal Transition Is Associated with a Distinct Tumor Microenvironment Including Elevation of Inflammatory Signals and Multiple Immune Checkpoints in Lung Adenocarcinoma. Clin Cancer Res 2016;22:3630-42.

59. Mak MP, Tong P, Diao L, et al. A Patient-Derived, PanCancer EMT Signature Identifies Global Molecular Alterations and Immune Target Enrichment Following Epithelial-to-Mesenchymal Transition. Clin Cancer Res 2016;22:609-20.

60. Cortez MA, Anfossi S, Ramapriyan R, et al. Role of miRNAs in immune responses and immunotherapy in cancer. Genes Chromosomes Cancer 2019;58:244-53.

61. Pardini B, Calin GA. MicroRNAs and Long Non-Coding RNAs and Their Hormone-Like Activities in Cancer. Cancers (Basel) 2019;11:378.

62. Zangari J, Ilie M, Rouaud F, et al. Rapid decay of engulfed extracellular miRNA by XRN1 exonuclease promotes transient epithelial-mesenchymal transition. Nucleic Acids Res 2018;46:3813-14.

63. Pasini L, Ulivi P. Extracellular Vesicles in Non-SmallCell Lung Cancer: Functional Role and Involvement in Resistance to Targeted Treatment and Immunotherapy. Cancers (Basel) 2019;12:40.

64. Steele KE, Brown C. Multiplex Immunohistochemistry for Image Analysis of Tertiary Lymphoid Structures in Cancer. Methods Mol Biol 2018;1845:87-98.

65. Thommen DS, Schreiner J, Müller P, et al. Progression of Lung Cancer Is Associated with Increased Dysfunction of T Cells Defined by Coexpression of Multiple Inhibitory Receptors. Cancer Immunol Res 2015;3:1344-55.

66. Goc J, Germain C, Vo-Bourgais TK, et al. Dendritic cells in tumor-associated tertiary lymphoid structures signal a Th1 cytotoxic immune contexture and license the positive prognostic value of infiltrating CD8+ T cells. Cancer Res 2014;74:705-15.

67. Inoue $\mathrm{Y}$, Yoshimura K, Kurabe N, et al. Prognostic impact of CD73 and A2A adenosine receptor expression in nonsmall-cell lung cancer. Oncotarget 2017;8:8738-51.

68. Mediavilla-Varela M, Castro J, Chiappori A, et al. A Novel Antagonist of the Immune Checkpoint Protein Adenosine A2a Receptor Restores Tumor-Infiltrating Lymphocyte Activity in the Context of the Tumor Microenvironment. Neoplasia 2017;19:530-6.

69. Vigano S, Alatzoglou D, Irving M, et al. Targeting Adenosine in Cancer Immunotherapy to Enhance T-Cell
Function. Front Immunol 2019;10:925.

70. Di Virgilio F, Adinolfi E. Extracellular purines, purinergic receptors and tumor growth. Oncogene 2017;36:293-303.

71. Di Virgilio F, Sarti AC, Falzoni S, et al. Extracellular ATP and P2 purinergic signalling in the tumour microenvironment. Nat Rev Cancer 2018;18:601-8.

72. Adinolfi E, De Marchi E, Orioli E, et al. Role of the P2X7 receptor in tumor-associated inflammation. Curr Opin Pharmacol 2019;47:59-64.

73. Cesaro A, Brest P, Hofman V, et al. Amplification loop of the inflammatory process is induced by $\mathrm{P} 2 \mathrm{X} 7 \mathrm{R}$ activation in intestinal epithelial cells in response to neutrophil transepithelial migration. Am J Physiol Gastrointest Liver Physiol 2010;299:G32-42.

74. De Marchi E, Orioli E, Pegoraro A, et al. The $\mathrm{P} 2 \mathrm{X} 7$ receptor modulates immune cells infiltration, ectonucleotidases expression and extracellular ATP levels in the tumor microenvironment. Oncogene 2019;38:3636-50.

75. Hofman P, Cherfils-Vicini J, Bazin M, et al. Genetic and pharmacological inactivation of the purinergic P2RX7 receptor dampens inflammation but increases tumor incidence in a mouse model of colitis-associated cancer. Cancer Res 2015;75:835-45.

76. O'Brien SM, Klampatsa A, Thompson JC, et al. Function of Human Tumor-Infiltrating Lymphocytes in EarlyStage Non-Small Cell Lung Cancer. Cancer Immunol Res 2019;7:896-909.

77. Thelen M, Lechner A, Wennhold K, et al. CD39 Expression Defines Cell Exhaustion in Tumor-Infiltrating CD8+ T Cells-Letter. Cancer Res 2018;78:5173-4.

78. Tøndell A, Wahl SGF, Sponaas AM, et al. Ectonucleotidase CD39 and Checkpoint Signalling Receptor Programmed Death 1 are Highly Elevated in Intratumoral Immune Cells in Non-small-cell Lung Cancer. Transl Oncol 2020;13:17-24.

79. Hofman V, Ilie M, Long E, et al. Detection of circulating tumor cells from lung cancer patients in the era of targeted therapy: promises, drawbacks and pitfalls. Curr Mol Med 2014;14:440-56.

80. Garrido-Navas C, de Miguel-Perez D, ExpositoHernandez J, et al. Cooperative and Escaping Mechanisms between Circulating Tumor Cells and Blood Constituents. Cells 2019;8:1382.

81. Heeke S, Mograbi B, Alix-Panabières C, et al. Never Travel Alone: The Crosstalk of Circulating Tumor Cells and the Blood Microenvironment. Cells 2019;8:714.

82. Wang WC, Zhang XF, Peng J, et al. Survival Mechanisms and Influence Factors of Circulating Tumor Cells. Biomed 
Res Int 2018;2018:6304701.

83. Mohme M, Riethdorf S, Pantel K. Circulating and disseminated tumour cells - mechanisms of immune surveillance and escape. Nat Rev Clin Oncol 2017;14:155-67.

84. Szczerba BM, Castro-Giner F, Vetter M, et al. Neutrophils escort circulating tumour cells to enable cell cycle progression. Nature 2019;566:553-7.

85. Hofman P, Badoual C, Henderson F, et al. Multiplexed Immunohistochemistry for Molecular and Immune Profiling in Lung Cancer-Just About Ready for PrimeTime? Cancers (Basel) 2019;11:283.

86. Parra ER, Francisco-Cruz A, Wistuba II. State-of-the-Art of Profiling Immune Contexture in the Era of Multiplexed Staining and Digital Analysis to Study Paraffin Tumor Tissues. Cancers (Basel) 2019;11:247.

87. Francisco-Cruz A, Parra ER, Tetzlaff MT, et al. Multiplex Immunofluorescence Assays. Methods Mol Biol 2020;2055:467-95.

88. Ilié M, Beaulande M, Ben Hadj S, et al. Chromogenic Multiplex Immunohistochemistry Reveals Modulation of the Immune Microenvironment Associated with Survival in Elderly Patients with Lung Adenocarcinoma. Cancers (Basel) 2018;10:326.

89. Kalra J, Baker J. Multiplex Immunohistochemistry for Mapping the Tumor Microenvironment. Methods Mol Biol 2017;1554:237-51.

90. Ilie M, Beaulande M, Hamila M, et al. Automated chromogenic multiplexed immunohistochemistry assay for diagnosis and predictive biomarker testing in non-small cell lung cancer. Lung Cancer 2018;124:90-4;

91. Morse MA, Osada T, Hobeika A, et al. Biomarkers and correlative endpoints for immunotherapy trials. Am Soc Clin Oncol Educ Book 2013. doi: 10.1200/EdBook_ AM.2013.33.e287.

92. Pockley AG, Foulds GA, Oughton JA, et al. Immune Cell Phenotyping Using Flow Cytometry. Curr Protoc Toxicol 2015;66:18.8.1-34.

93. Lavin Y, Kobayashi S, Leader A, et al. Innate Immune Landscape in Early Lung Adenocarcinoma by Paired Single-Cell Analyses. Cell 2017;169:750-765.e17.

94. Damotte D, Warren S, Arrondeau J, et al. The tumor inflammation signature (TIS) is associated with anti-PD-1 treatment benefit in the CERTIM pan-cancer cohort. J Transl Med 2019;17:357.

95. Ståhl PL, Salmén F, Vickovic S, et al. Visualization and analysis of gene expression in tissue sections by spatial transcriptomics. Science 2016;353:78-82
96. Guo X, Zhang Y, Zheng L, et al. Global characterization of $\mathrm{T}$ cells in non-small-cell lung cancer by single-cell sequencing. Nat Med 2018;24:978-85.

97. Stubbington MJT, Rozenblatt-Rosen O, Regev A, et al. Single-cell transcriptomics to explore the immune system in health and disease. Science 2017;358:58-63.

98. Vegh P, Haniffa M. The impact of single-cell RNA sequencing on understanding the functional organization of the immune system. Brief Funct Genomics 2018;17:265-72.

99. Dafni U, Tsourti Z, Vervita K, et al. Immune checkpoint inhibitors, alone or in combination with chemotherapy, as first-line treatment for advanced non-small cell lung cancer. Asystematic review and network meta-analysis. Lung Cancer 2019;134:127-40.

100. Gandhi L, Rodríguez-Abreu D, Gadgeel S, et al. KEYNOTE-189 Investigators. Pembrolizumab plus Chemotherapy in Metastatic Non-Small-Cell Lung Cancer. N Engl J Med 2018;378:2078-92.

101.Hellmann MD, Paz-Ares L, Bernabe Caro R, et al Nivolumab plus Ipilimumab in Advanced Non-Small-Cell Lung Cancer. N Engl J Med 2019;381:2020-31.

102.Paz-Ares L, Luft A, Vicente D, et al. Pembrolizumab plus Chemotherapy for Squamous Non-Small-Cell Lung Cancer. KEYNOTE-407 Investigators. N Engl J Med 2018;379:2040-51

103. Wang C, Qiao W, Jiang Y, et al. The landscape of immune checkpoint inhibitor plus chemotherapy versus immunotherapy for advanced non-small-cell lung cancer: A systematic review and meta-analysis. J Cell Physiol 2020;235:4913-27.

104.Aspeslagh S, Postel-Vinay S, Rusakiewicz S, et al. Rationale for anti-OX40 cancer immunotherapy. Eur J Cancer 2016;52:50-66.

105. Chesney JA, Mitchell RA, Yaddanapudi K. Myeloidderived suppressor cells-a new therapeutic target to overcome resistance to cancer immunotherapy. J Leukoc Biol 2017;102:727-40.

106. Clara JA, Monge C, Yang Y, et al. Targeting signalling pathways and the immune microenvironment of cancer stem cells - a clinical update. Nat Rev Clin Oncol 2020;17:204-32.

107. Demaria O, Cornen S, Daëron M, et al. Harnessing innate immunity in cancer therapy. Nature 2019;574:45-56.

108. De Matteis S, Canale M, Verlicchi A, et al. Advances in Molecular Mechanisms and Immunotherapy Involving the Immune Cell-Promoted Epithelial-to-Mesenchymal Transition in Lung Cancer. J Oncol 2019;2019:7475364. 109.Leone RD, Emens LA. Targeting adenosine for cancer 
immunotherapy. J Immunother Cancer 2018;6:57.

110.Li XY, Moesta AK, Xiao C, et al. Targeting CD39 in Cancer Reveals an Extracellular ATP- and InflammasomeDriven Tumor Immunity. Cancer Discov 2019;9:1754-73.

111. Anfray C, Ummarino A, Andón FT, et al. Current Strategies to Target Tumor-Associated-Macrophages to Improve Anti-Tumor Immune Responses. Cells 2019;9:46.

112. Gonzalez-Aparicio M, Alfaro C. Significance of the IL-8 pathway for immunotherapy. Hum Vaccin Immunother 2019. doi: 10.1080/21645515.2019.1696075.

113. Cassetta L, Pollard JW. Targeting macrophages: therapeutic approaches in cancer. Nat Rev Drug Discov 2018;17:887-904.

114. Cortese N, Donadon M, Rigamonti A, et al. Macrophages at the crossroads of anticancer strategies. Front Biosci (Landmark Ed) 2019;24:1271-83.

115.Shaul ME, Fridlender ZG. Tumour-associated neutrophils in patients with cancer. Nat Rev Clin Oncol 2019;16:601-20.

116. Di Vito C, Mikulak J, Zaghi E, et al. NK cells to cure cancer. Semin Immunol 2019;41:101272.

117. Tang H, Qiu X, Timmerman C, et al. Targeting Tertiary Lymphoid Structures for Tumor Immunotherapy. Methods Mol Biol 2018;1845:275-86.

118. Shabbir M, Thompson C, Jarmulowiczc M, et al. Effect of extracellular ATP on the growth of hormone-refractory prostate cancer in vivo. BJU Int 2008;102:108-12.

119. Wang Y, Sun Y, Li D, et al. Platelet P2Y12 is involved in murine pulmonary metastasis. PLoS One 2013;8:e80780

120.Liu Y, Liu L. The pro-tumor effect and the anti-tumor effect of neutrophils extracellular traps. Biosci Trends 2020;13:469-75

121. Falk AT, Yazbeck N, Guibert N, et al. Effect of mutant variants of the KRAS gene on PD-L1 expression and on the immune microenvironment and association with clinical outcome in lung adenocarcinoma patients. Lung Cancer 2018;121:70-5.

122.Mansuet-Lupo A, Alifano M, Pécuchet N, et al. Intratumoral Immune Cell Densities Are Associated with Lung Adenocarcinoma Gene Alterations. Am J Respir Crit Care Med 2016;194:1403-12.

123. Chen N, Fang W, Lin Z, et al. KRAS mutation-induced upregulation of PD-L1 mediates immune escape in human lung adenocarcinoma. Cancer Immunol Immunother 2017:1175-87.

124. Skoulidis F, Byers LA, Diao L, et al. Co-occurring genomic alterations define major subsets of KRAS-mutant lung adenocarcinoma with distinct biology, immune profiles, and therapeutic vulnerabilities. Cancer Discov 2015;5:860-77.

125. Dias Carvalho P, Machado AL, Martins F, et al. Targeting the Tumor Microenvironment: An Unexplored Strategy for Mutant KRAS Tumors. Cancers (Basel) 2019;11:2010.

126. Hallin J, Engstrom LD, Hargis L, et al. The KRASG12C Inhibitor MRTX849 Provides Insight toward Therapeutic Susceptibility of KRAS-Mutant Cancers in Mouse Models and Patients. Cancer Discov 2020;10:54-71.

127.Janes MR, Zhang J, Li LS, et al. Targeting KRAS Mutant Cancers with a Covalent G12C-Specific Inhibitor. Cell 2018;172:578-589.e17.

128. Molina-Arcas M, Moore C, Rana S, et al. Development of combination therapies to maximize the impact of KRAS-G12C inhibitors in lung cancer. Sci Transl Med 2019;11:eaaw7999.

129. Bera K, Schalper KA, Rimm DL, et al. Artificial intelligence in digital pathology - new tools for diagnosis and precision oncology. Nat Rev Clin Oncol 2019;16:703-15.

130.Wang S, Rong R, Yang DM, et al. Computational staining of pathology images to study the tumor microenvironment in lung cancer. Cancer Res 2020;80:2056-66.
Cite this article as: Hofman P. New insights into the interaction of the immune system with non-small cell lung carcinomas. Transl Lung Cancer Res 2020;9(5):2199-2213. doi: 10.21037/tlcr-20-178 